\title{
DFT explorations of quadrupole coupling constants for planar 5-fluorouracil pairs
}

\author{
Mahmoud Mirzaei ${ }^{\text {a,*}}$, Oğuz Gülseren ${ }^{\mathrm{b}}$, Nasser Hadipour ${ }^{\mathrm{c}}$ \\ a Bioinformatics Research Center, School of Pharmacy and Pharmaceutical Sciences, Isfahan University of Medical Sciences, Isfahan, Iran \\ ${ }^{\mathrm{b}}$ Department of Physics, Faculty of Science, Bilkent University, Ankara, Turkey \\ ${ }^{\mathrm{c}}$ Department of Chemistry, Faculty of Science, Tarbiat Modares University, Tehran, Iran
}

\section{A R T I C L E I N F O}

\section{Article history:}

Received 2 March 2016

Received in revised form 6 June 2016

Accepted 6 June 2016

Available online 6 June 2016

\section{Keywords:}

Fluorouracil

Hydrogen bond

Density functional theory

Quadrupole coupling constant

\begin{abstract}
A B S T R A C T
Atomic scale properties of quadrupole coupling constants $\left(C_{\mathrm{Q}}\right)$ have been evaluated for singular and paired 5-fluorouracil (FU) models. Structural possibilities and properties for various types of hydrogen bonded (HB) homo pairs of FU have been investigated based on density functional theory (DFT) calculations. The models have been optimized to obtain the minimum energy level structures and only the planar molecular pairs have been considered. Various types of $\mathrm{HB}$ interactions have also managed the molecular shapes for the FU pairs. Different types of energies and also electron transferring properties have been investigated by the evaluated optimized properties. The atomic scale results indicated different strengths of $\mathrm{HB}$ interactions for FU pairs according to the changes of $C_{\mathrm{Q}}$ properties for atoms in the singular and paired systems depending on the strength of interactions.
\end{abstract}

(c) 2016 Elsevier B.V. All rights reserved.

\section{Introduction}

Uracil, the characteristic nucleobase of ribonucleic acid (RNA), is capable of contributing to hydrogen bonding (HB) interactions with another uracil or other molecules [1,2]. It is well know that the $\mathrm{HB}$ interactions are among the most important inter- and intra-molecular interactions especially in living biological systems [3]. Several interactions between biological molecules could be taken place because of $\mathrm{HB}$ interactions, so $\mathrm{HB}$ is critical for the building blocks of life [4]. The presence of HB interactions is also essential for the proper ligand - receptor connections in drug deliveries [5]. Furthermore, several specific chemical and biochemical systems exist because of the strong and weak HB interactions between molecular counterparts [6,7]. In addition to HB interactions between hetero molecular counterparts, the properties for HB interactions between homo molecules of nucleobases are also important and they are worth to investigate [8-10]. In real systems, the first candidate for a nucleobase to interact is another nucleobase molecule of the same type in a homo pair interacting system [11]. The possibilities of existence and properties of homo base pairs of uracil have been investigated by earlier studies [1215]. In fact, the effects of HB interactions on the original properties of a molecule could be systematically investigated in paired systems, simply avoiding the complexity of multi component systems

\footnotetext{
* Corresponding author.

E-mail address: mdmirzaei@pharm.mui.ac.ir (M. Mirzaei).
}

[16]. The number of homo interacting uracil molecules could be increased as dimer, trimer, and tetramer, up to polymers defining their amorphous or crystalline structures [17]. Classical HBs are defined between two atoms of nitrogen, oxygen, and fluorine electronegative atoms, but they could also exist between carbon atoms as a non-classical type [18]. It is important to note that the weak interactions are dominant to predict the three-dimensional structures of the complex systems [19]. In complementary to experimental measurements, quantum chemical calculations could yield insightful information for the weak HBs properties in paired systems [20]. As a matter of fact, computational results could be considered either prior to the experiments to predict and/or after the experiments to understand and interpret the molecular systems $[21,22]$.

Within this work, possible molecular pairs of 5-fluorouracil (FU) (Figs. 1-3) through $\mathrm{HB}$ interactions are investigated from firstprinciples calculations based on density functional theory (DFT). FU, which has been used for therapies of various types of cancers for a long time, is a carbon-five fluorinated derivative of uracil nucleobase [23]. FU is an efficient drug for cancer therapies, but several side effects were reported for the patients using this drug [24]. Therefore, characterization and understanding of the electronic and structural properties of FU is still an appealing topic of research [25-27]. Parallel to original uracil, FU molecules could also contribute to classical and non-classical HB interactions to make FU pairs. Using this possibility, we investigated various types of the $\mathrm{HB}$ interacted pairs of FU including $\mathrm{C}=\mathrm{O} \cdots \mathrm{H}-\mathrm{N}, \mathrm{C}=\mathrm{O} \cdots \mathrm{H}-\mathrm{C}$, 


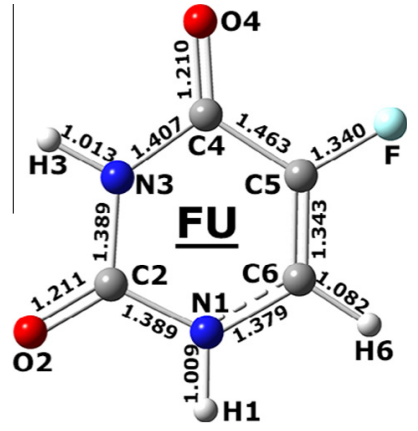

Fig. 1. The optimized geometry of the lone 5-fluorouracil (FU).

$\mathrm{C}-\mathrm{F} \cdots \mathrm{H}-\mathrm{N}$, and $\mathrm{C}-\mathrm{F} \cdots \mathrm{H}-\mathrm{C}$ interactions. The major goal of this work is to characterize the stable configurations as well as the properties of homo pairs of FU (Figs. 2 and 3) to recognize the effects of HB interactions on the atomic and molecular level properties of FU. To this aim, various parameters (Tables 1-4) have been evaluated for lone and paired FU structures including the different conditions of paired FU structures in order to compare HB effects on the original properties.

\section{Computational details}

DFT computations of this work have been done employing the B3LYP exchange-correlation functional and the $6-311++G^{* *}$ stan-

(a)

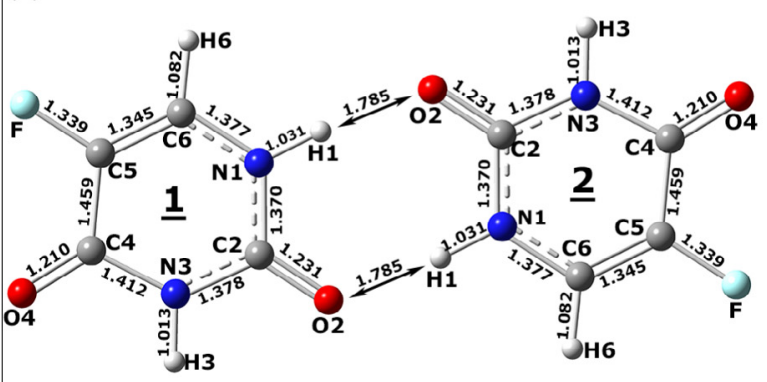

(b)

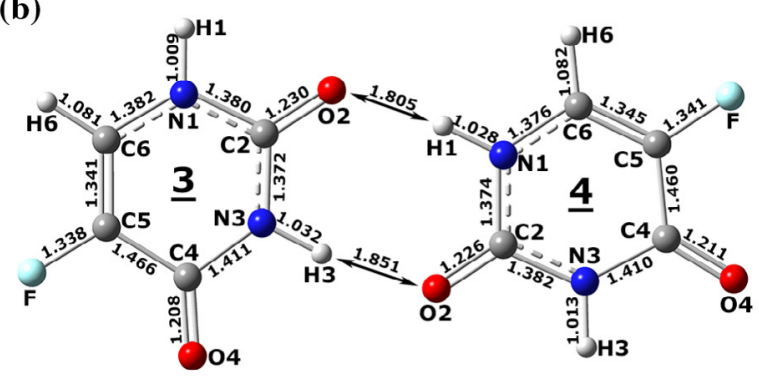

(c)

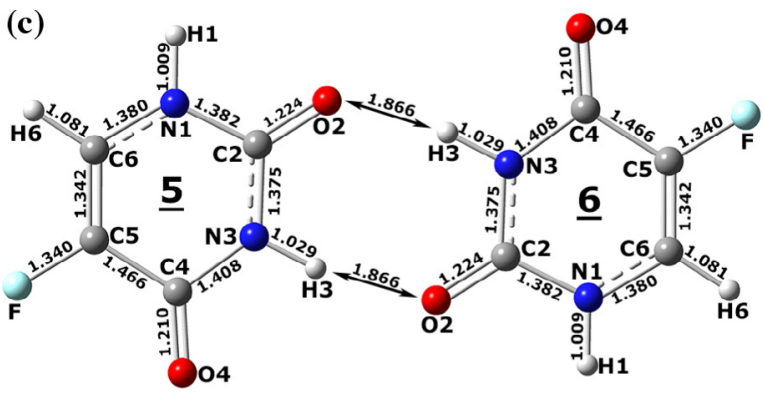

dard basis set as implemented in the Gaussian 98 package [28]. The investigated models are the singular FU (Fig. 1) and all possible HB interacted homo molecular pairs (Figs. 2 and 3). Two sets of FU pairs have been constructed including only $\mathrm{O} \cdots \mathrm{H}$ interactions for the first set (Fig. 2) and $\mathrm{O} \cdots \mathrm{H} / \mathrm{F} \cdots \mathrm{H}$ interactions for the second set (Fig. 3). Note that all possibilities for making homo molecular pairs have been considered to make the FU pairs in eleven available forms (Figs. 2 and 3). The model structures including singular FU and various pairs have been optimized to obtain the corresponding minimum energy structures, in which the optimized structures and bond lengths are exhibited in the panels of Figs. 1-3. To confirm the global minimum points, frequency calculations have been also performed for the optimized structures; all structures have been approved. Even though the $6-311++G^{* *}$ is a large-size basis set, the basis set superposition error (BSSE) has been still examined for the models $[29,30]$, and the results show that the changes of magnitudes of energies are almost negligible. The properties of optimized structures including total energies $\left(E_{\mathrm{T}}\right)$, binding energies $\left(E_{\mathrm{B}}\right)$, energy levels for the highest occupied and the lowest unoccupied molecular orbitals ( $E_{\mathrm{HOMO}}$ and $E_{\mathrm{LUMO}}$ ), magnitudes of energy differences for receiving and releasing one electron $\left(\Delta E_{\mathrm{ET}-}\right.$ and $\left.\Delta E_{\mathrm{ET}+}\right)$, and dipole moments $\left(D_{\mathrm{M}}\right)$ are listed in Table 1 . The magnitudes for total energies, molecular orbital energies, and dipole moments have been directly obtained from computational results. To evaluate the binding energies, the energy differences between the obtained total energy of each pair and the duplicated energy of singular FU have been used. For energy differences in one electron transferring, the single point energies have been calculated for

(d)
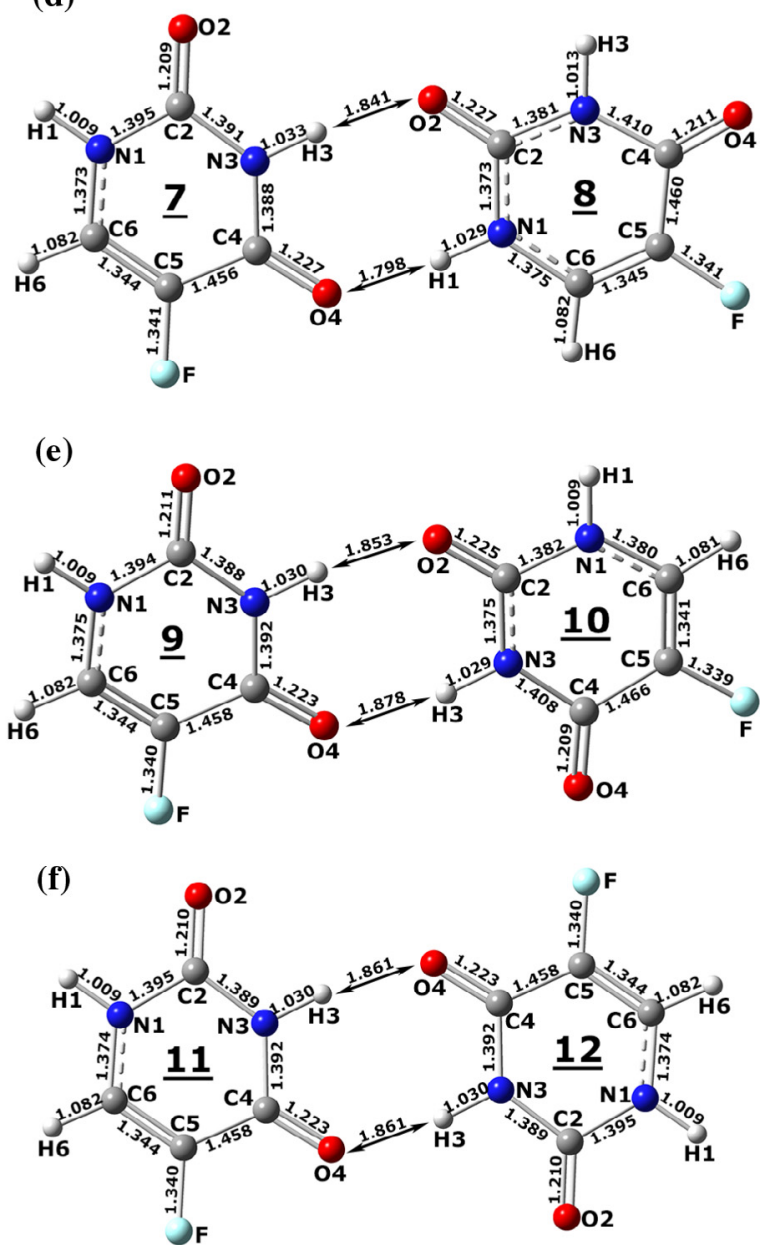

Fig. 2. The optimized geometries of the FU pairs through $\mathrm{C}=\mathrm{O} \cdots \mathrm{H}-\mathrm{N}$ hydrogen bonds; panels a-f. 
(g)
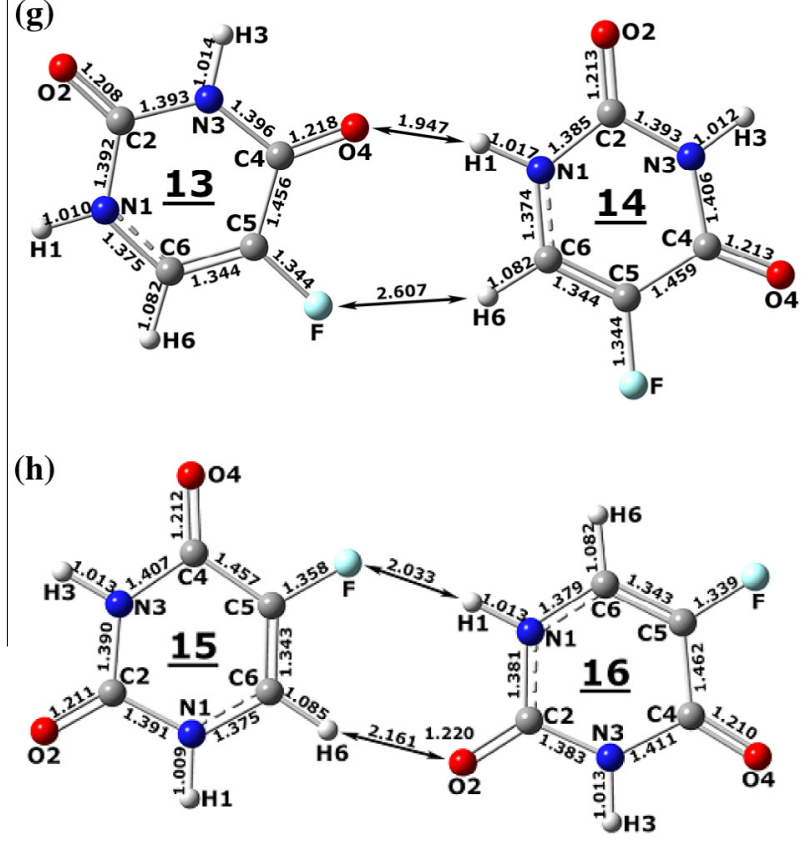
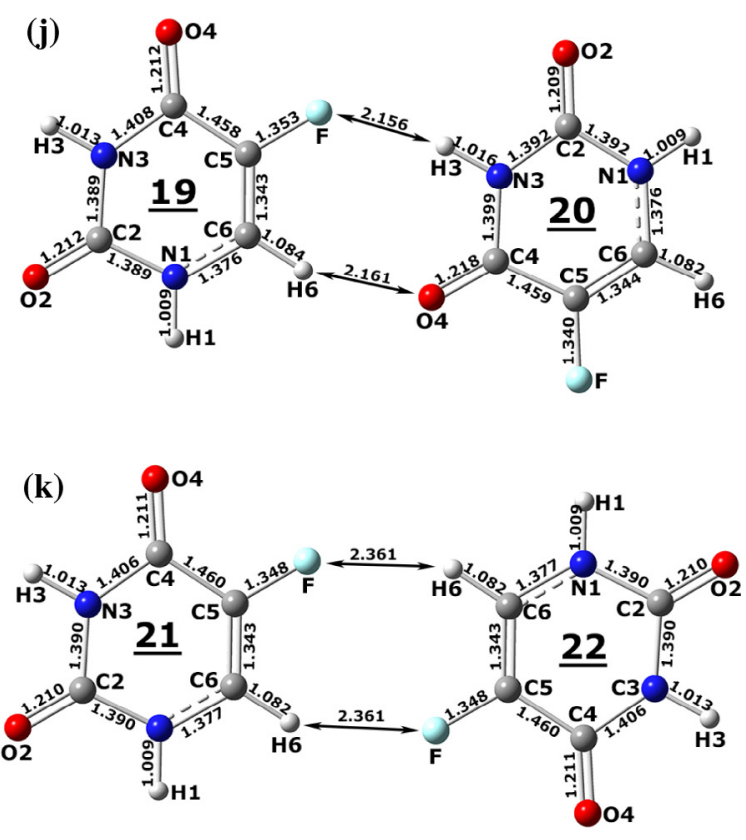

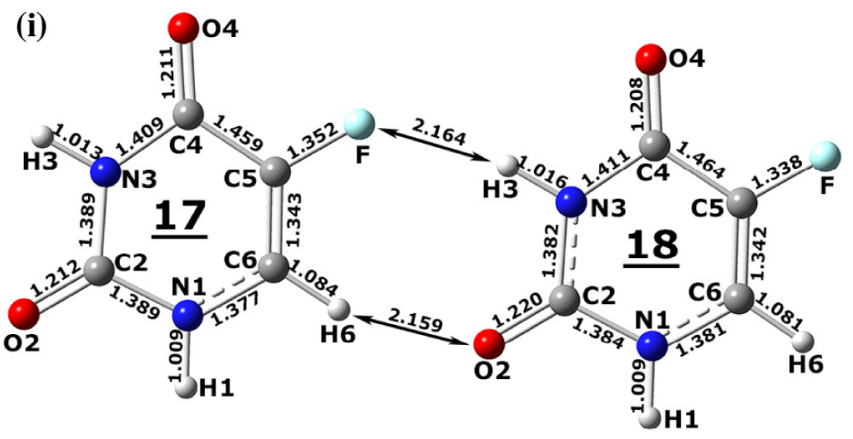

Fig. 3. The optimized geometries of the FU pairs through $\mathrm{C}-\mathrm{F} \cdots \mathrm{H}-\mathrm{N}, \mathrm{C}-\mathrm{F} \cdots \mathrm{H}-\mathrm{C}, \mathrm{C}=\mathrm{O} \cdots \mathrm{H}-\mathrm{C}$, and $\mathrm{C}=\mathrm{O} \cdots \mathrm{H}-\mathrm{N}$ hydrogen bonds; panels $\mathrm{g}-\mathrm{k}$.

Table 1

Binding energies $\left(E_{\mathrm{B}}\right)$, energy levels for the highest occupied and the lowest unoccupied molecular orbitals ( $E_{\mathrm{HOMO}}$ and $E_{\mathrm{LUMO}}$ ), magnitudes of energy differences for receiving and releasing one electron $\left(\Delta E_{\mathrm{ET}-}\right.$ and $\left.\Delta E_{\mathrm{ET}+}\right)$, (all in eV) and dipole moments $\left(D_{\mathrm{M}}\right)$ of optimized structures. ${ }^{\mathrm{a}}$

\begin{tabular}{lllllll}
\hline Model & $E_{\mathrm{B}}$ & $E_{\mathrm{HOMO}}$ & $E_{\mathrm{LUMO}}$ & $\Delta E_{\mathrm{ET}-}$ & $\Delta E_{\mathrm{ET}+}$ & \multicolumn{1}{c}{$D_{\mathrm{M}}$} \\
\hline Monomer & - & -7.322 & -1.993 & 0.074 & 9.508 & 4.214 \\
(a) $1 \cdots 2$ & -0.816 & -7.364 & -2.076 & -0.609 & 8.910 & 0.003 \\
(b) $3 \cdots 4$ & -0.648 & -7.174 & -2.057 & -0.497 & 8.835 & 8.266 \\
(c) $5 \cdots 6$ & -0.525 & -7.268 & -1.882 & -0.416 & 8.802 & 0.006 \\
(d) $7 \cdots 8$ & -0.679 & -7.127 & -2.243 & -0.584 & 8.796 & 7.570 \\
(e) $9 \cdots 10$ & -0.520 & -7.217 & -1.983 & -0.461 & 8.747 & 4.958 \\
(f) $11 \cdots 12$ & -0.523 & -7.168 & -2.013 & -0.511 & 8.697 & 0.002 \\
(g) $13 \cdots 14$ & -0.351 & -6.723 & -2.709 & -0.799 & 8.677 & 10.172 \\
(h) $15 \cdots 16$ & -0.326 & -7.432 & -2.138 & -0.623 & 8.977 & 0.986 \\
(i) $17 \cdots 18$ & -0.239 & -7.193 & -2.153 & -0.554 & 8.862 & 8.562 \\
(j) $19 \cdots 20$ & -0.263 & -7.151 & -2.265 & -0.625 & 8.831 & 7.284 \\
(k) $21 \cdots 22$ & -0.153 & -7.447 & -2.143 & -0.668 & 8.972 & 0.063 \\
\hline
\end{tabular}

a See Figs. 1-3 for the model structures.

the ionic forms and then the energy differences between the ionic and neutral forms have been calculated.

To better recognize the changes of properties for the investigated FU pairs through $\mathrm{HB}$ interactions, quadrupole coupling constants $\left(C_{\mathrm{Q}}\right)$ have been evaluated for each atom of the optimized singular FU and paired structures (Tables 3 and 4). The magnitudes of $C_{\mathrm{Q}}$ imply for the interaction energies between nuclear electric
Table 2

Quadrupole coupling constants for $\mathrm{N}$ and $\mathrm{O}$ atoms of uracil and 2-thiouracil $\left(C_{\mathrm{Q}} / \mathrm{MHz}\right)$.

\begin{tabular}{lllll}
\hline Model & $\mathrm{N}_{1}$ & $\mathrm{~N}_{3}$ & $\mathrm{O}_{2}$ & $\mathrm{O}_{4}$ \\
\hline Uracil $^{\mathrm{a}}$ & 4.04 & 3.80 & 8.57 & 9.79 \\
Exp. $_{\text {Comput. }}$ & $3.74^{\mathrm{b}}$ & $3.74^{\mathrm{b}}$ & $7.61^{\mathrm{c}}$ & $7.85^{\mathrm{c}}$ \\
2-Thiouracil $^{\mathrm{a}}$ & $3.89^{\mathrm{d}}$ & $3.56^{\mathrm{d}}$ & $8.60^{\mathrm{c}}$ & $9.82^{\mathrm{c}}$ \\
Exp. $^{\mathrm{e}}$ & 3.70 & 3.47 & & 9.80 \\
Comput. $^{\mathrm{e}}$ & 3.41 & 3.12 & & \\
\hline
\end{tabular}

a This work, B3LYP/6-311++G** computations.

b Ref. [38].

c Ref. [2], B3LYP/6-311++G** computations.

d Ref. [37], equilibrium values, (all)CCSD(T)/wCVTZ computations.

e Ref. [35], equilibrium values, CCSD(T)/cc-pVTZ computations.

quadrupole moment $(Q)$ and electric field gradient (EFG) tensors $\left(\left|q_{\mathrm{zz}}\right|>\left|q_{\mathrm{yy}}\right|>\left|q_{\mathrm{xx}}\right|\right)$ at the electronic sites of atoms [31]. To calculate the exact magnitudes for $C_{\mathrm{Q}}$, the equation $C_{\mathrm{Q}}(\mathrm{MHz})=e^{2} \mathrm{Q} q_{\mathrm{zz}} h^{-1}$ is used, in which the standard values for $Q$ are employed [32]. The $C_{Q}$ properties could yield insightful information about the characteristics of matters because the originated EFG tensors from the electronic sites of atoms could indicate the effect of any perturbation $[33,34]$. Electrons are partially shared between atoms to make HB interactions; therefore, the $C_{Q}$ properties could determine the atomic contributions to the HB interactions [33,34]. The magnitudes of $C_{\mathrm{Q}}$ could be experimentally measured by nuclear quadrupole resonance $(\mathrm{NQR})$ spectroscopy and they could be also 
Table 3

Quadrupole coupling constants for $\mathrm{H}$ and $\mathrm{C}$ atoms $\left(C_{\mathrm{Q}} / \mathrm{kHz}\right){ }^{\mathrm{a}}$

\begin{tabular}{llllllll}
\hline Model & $\mathrm{H}_{1}$ & $\mathrm{H}_{3}$ & $\mathrm{H}_{6}$ & $\mathrm{C}_{2}$ & $\mathrm{C}_{4}$ & $\mathrm{C}_{5}$ & $\mathrm{C}_{6}$ \\
\hline Monomer & 269 & 262 & 212 & 1970 & 2498 & 3444 & 2264 \\
(a) $1 \cdots 2$ & 197 & 261 & 210 & 2116 & 2529 & 3425 & 2313 \\
& {$[197]$} & {$[261]$} & {$[210]$} & {$[2116]$} & {$[2529]$} & {$[3425]$} & {$[2313]$} \\
(b) $3 \cdots 4$ & 269 & 202 & 212 & 2119 & 2456 & 3356 & 2254 \\
& {$[205]$} & {$[261]$} & {$[211]$} & {$[2094]$} & {$[2497]$} & {$[3474]$} & {$[2274]$} \\
(c) $5 \cdots 6$ & 269 & 209 & 212 & 2094 & 2485 & 3411 & 2244 \\
& {$[269]$} & {$[209]$} & {$[212]$} & {$[2094]$} & {$[2485]$} & {$[3411]$} & {$[2244]$} \\
(d) $7 \cdots 8$ & 268 & 200 & 211 & 2002 & 2723 & 3504 & 1674 \\
& {$[204]$} & {$[261]$} & {$[211]$} & {$[2098]$} & {$[2504]$} & {$[3474]$} & {$[2295]$} \\
(e) $9 \cdots 10$ & 269 & 207 & 211 & 1992 & 2679 & 3471 & 2275 \\
& {$[269]$} & {$[209]$} & {$[212]$} & {$[2099]$} & {$[2472]$} & {$[3405]$} & {$[2238]$} \\
(f) $11 \cdots 12$ & 269 & 207 & 211 & 1998 & 2685 & 3489 & 2274 \\
& {$[269]$} & {$[207]$} & {$[211]$} & {$[1998]$} & {$[2685]$} & {$[3489]$} & {$[2274]$} \\
(g) $13 \cdots 14$ & 267 & 259 & 210 & 1989 & 2366 & 2378 & 2359 \\
& {$[238]$} & {$[263]$} & {$[203]$} & {$[1980]$} & {$[2526]$} & {$[3594]$} & {$[2313]$} \\
(h) $15 \cdots 16$ & 268 & 262 & 190 & 1981 & 2498 & 4079 & 2755 \\
& {$[252]$} & {$[261]$} & {$[211]$} & {$[2048]$} & {$[2502]$} & {$[3406]$} & {$[2320]$} \\
(i) $17 \cdots 18$ & 269 & 262 & 193 & 1977 & 2494 & 3872 & 2718 \\
& {$[269]$} & {$[248]$} & {$[212]$} & {$[2047]$} & {$[2464]$} & {$[3374]$} & {$[2271]$} \\
(j) $19 \cdots 20$ & 269 & 262 & 193 & 1977 & 2499 & 3894 & 2733 \\
& {$[268]$} & {$[248]$} & {$[211]$} & {$[1987]$} & {$[2620]$} & {$[3476]$} & {$[2301]$} \\
(k) $21 \cdots 22$ & 268 & 262 & 206 & 1979 & 2503 & 3766 & 2446 \\
& {$[268]$} & {$[262]$} & {$[206]$} & {$[1979]$} & {$[2503]$} & {$[3766]$} & {$[2446]$} \\
\hline
\end{tabular}

a See Figs. 1-3 for the model structures and atoms numbers.

Table 4

Quadrupole coupling constants for $\mathrm{N}, \mathrm{O}$ and $\mathrm{F}$ atoms $\left(C_{\mathrm{Q}} / \mathrm{kHz}\right){ }^{\mathrm{a}}$

\begin{tabular}{llllll}
\hline Model & $\mathrm{N}_{1}$ & $\mathrm{~N}_{3}$ & $\mathrm{O}_{2}$ & $\mathrm{O}_{4}$ & $\mathrm{~F}$ \\
\hline Monomer & 4281 & 3852 & 8495 & 9775 & 68,019 \\
(a) $1 \cdots 2$ & 3441 & 3736 & 7950 & 9731 & 67,957 \\
& {$[3441]$} & {$[3736]$} & {$[7950]$} & {$[9731]$} & {$[67,957]$} \\
(b) $3 \cdots 4$ & 4235 & 3178 & 8028 & 10,039 & 67,797 \\
& {$[3518]$} & {$[3754]$} & {$[8129]$} & {$[9725]$} & {$[67,895]$} \\
(c) $5 \cdots 6$ & 4233 & 3234 & 8198 & 9978 & 67,771 \\
& {$[4233]$} & {$[3234]$} & {$[8198]$} & {$[9978]$} & {$[67,771]$} \\
(d) $7 \cdots 8$ & 4138 & 3169 & 8699 & 9209 & 68,155 \\
& {$[3486]$} & {$[3752]$} & {$[8108]$} & {$[9715]$} & {$[67,899]$} \\
(e) $9 \cdots 10$ & 4188 & 3223 & 8644 & 9422 & 68,118 \\
& {$[4242]$} & {$[3239]$} & {$[8181]$} & {$[10,001]$} & {$[67,757]$} \\
(f) $11 \cdots 12$ & 4184 & 3225 & 8666 & 9393 & 68,112 \\
& {$[4184]$} & {$[3224]$} & {$[8666]$} & {$[9394]$} & {$[68,112]$} \\
(g) $13 \cdots 14$ & 4168 & 3780 & 8589 & 9497 & 67,852 \\
& {$[3761]$} & {$[3842]$} & {$[8566]$} & {$[9654]$} & {$[67,806]$} \\
(h) $15 \cdots 16$ & 4157 & 3865 & 8543 & 9684 & 66,820 \\
& {$[4001]$} & {$[3796]$} & {$[8362]$} & {$[9773]$} & {$[66,058]$} \\
(i) $17 \cdots 18$ & 4182 & 3864 & 8506 & 9716 & 67,260 \\
& {$[4260]$} & {$[3662]$} & {$[8365]$} & {$[9909]$} & {$[67,982]$} \\
(j) $19 \cdots 20$ & 4167 & 3864 & 8512 & 9704 & 67,245 \\
& {$[4205]$} & {$[3662]$} & {$[8601]$} & {$[9605]$} & {$[68,127]$} \\
(k) $21 \cdots 22$ & 4218 & 3856 & 8534 & 9724 & 73,668 \\
& {$[4218]$} & {$[3856]$} & {$[8534]$} & {$[9724]$} & {$[73,668]$} \\
\hline & & &
\end{tabular}

a See Figs. 1-3 for the model structures and atoms numbers.

reproduced by computations [31]. It is an advantage of high level quantum chemical calculations to produce reliable spectroscopic properties prior to experiments for predictions, or after the experimental achievements for better explanations of the results $[21,22]$. To validate the obtained results of this work, the values were compared with the achievements of parallel studies on the uracil-related compounds, in which the overall achievements are compatible $[2,13,14,35-37]$. The fluoride functional group makes FU as a different structure in comparison with uracil or other derivatives especially in the interacting systems; therefore, the results of monomer are preferred to be compared in this case. In an earlier work [14], the dependencies of $C_{\mathrm{Q}}$ properties of temperature of crystallization were investigated by DFT calculations, in which the values of $C_{Q}$ properties of atoms were significantly dif- ferent compering to structures at different temperatures. In order to validate our methodology, we compared the available calculated and experimental values of $C_{\mathrm{Q}}$ properties for uracil and 2-thiouracil with the B3LYP/6-311++G** results (Table 2). It is shown that $C_{\mathrm{Q}}$ of nitrogen atoms are closer to the experiments than those of oxygen atoms, which are overestimated by computations as mentioned before [2]. However, our equilibrium $C_{Q}$ parameters are similar to previously reported DFT ones for oxygen atoms of uracil [2], and agree well with CCSD (T) estimates for nitrogen atoms of uracil [37] and 2-thiouracil [35]. Since we are comparing the results with paired models, this overestimation could be somehow neglected as the amount of changes of magnitudes through paired structures are important for us. It is noted that the mission of computational studies in not only to regenerate the exact values of experimental properties, but also to interpret what is happening inside the complex systems which is the main aim of this work. In the case of reproducing the exact experimental parameters, the model system in computations could be considered as much similar to real environment as possible in addition to a good theoretical method. In addition to employed theoretical levels, crystallographic information, investigating possible positions for other molecules around the target one, and solvent media are all very much important to reproduce better comparable parameters with the experiments $[2,9,29]$. In some cases, the computed results could also help to understand what happens in the real systems by modifying the molecular system of exploring crystalline effects [39]. However, in all cases, having the standard theoretical methodologies are important to achieve the purpose.

\section{Results and discussion}

The results of computations on singular FU (Fig. 1) and two sets of paired molecules (Figs. 2 and 3) are summarized in Tables 1-4 and the optimized bond lengths are directly exhibited for the corresponding bonds in the panels of figures. There are six atomic positions in FU possible for interactions with other molecules like another FU through HBs. Therefore, all possibilities of intermolecular interactions have been considered to make paired molecules of FU according to examining contributions of all atomic positions to the HB interactions. The amine nitrogen atoms and the keto oxygen atoms are the main positions for making strong HB interactions. Moreover, the fluorine atom and the hydrogen atom number six are the other possible positions for contributing to $\mathrm{HB}$ interactions. As a result, the paired structures are divided into two sets of pairs based on types of interactions; the first set includes only keto - amine interactions (Fig. 2) whereas the fluorine atoms and the hydrogen atom number six also participate in HB interactions in addition to keto - amine interactions in the second set (Fig. 3). The optimized geometries of paired molecules show that the intermolecular distance between two molecules is larger in the second set comparing with the first set. This is due to the strength of $\mathrm{HB}$ interactions and also the positions of participating atoms towards each other for contributing to interactions. The eleven considered FU pairs are all in planar system. According to the results of binding energies of Table 1, which are obtained by energy differences between the paired and singular FU structures, the most energetic binding is taken place for panel (a) and the least energetic one is for panel (k). Comparing the binding energies of the first and second sets of FU pairs indicates that the formations of the first set, which are dependent on $\mathrm{C}=\mathrm{O} \cdots \mathrm{H}-\mathrm{N}$ type of $\mathrm{HB}$ interactions, are more energetically favorable than the second set, which are dependent on $\mathrm{C}=\mathrm{O} \cdots \mathrm{H}-\mathrm{N}, \mathrm{C}=\mathrm{O} \cdots \mathrm{H}-\mathrm{C}, \mathrm{C}-\mathrm{F} \cdots \mathrm{H}-\mathrm{N}$, and $\mathrm{C}-\mathrm{F} \cdots \mathrm{H}-\mathrm{C}$ types of $\mathrm{HB}$ interactions. The strength of binding is reduced in the second set due to different types of participating $\mathrm{HB}$ interactions whereas the strong classical HB interactions are only 
managing the properties for the first set of FU pairs. The trend of the binding energies of two pairs set are in agreement with the longer intermolecular distances of two molecules in the second set in comparison with the first set.

The obtained energies of the molecular orbitals indicate that the energy levels are changed among the molecular systems. From singular FU to molecular pairs, both the levels of HOMO and LUMO and also the separations between the two levels are changed. In all pair cases, the separations between HOMO and LUMO are reduced in comparison to the singular FU. In order to estimate the one-electron transferring of FU structures, the energy differences between neutral and ionic forms have been evaluated for both singular FU and molecular pairs. The results indicated that receiving electron is unfavorable for singular FU but it is improved in the molecular pair cases as could be seen by the positive magnitude of energy difference for singular FU, but negative ones for the paired molecules. Different behavior is observed in the magnitudes of energy differences among anionic and cationic forms of the investigated molecular pairs. Interestingly, the energy difference magnitude for the cationic form is reduced in the pairs comparing with the singular molecule. Different polarities are also observed for the paired systems according the molecular orientations and the participating atoms in the HB interactions. As highlighted remarks of this section, it could be mentioned that the intra- and inter-molecular geometries of FU structure have been changed during the paired interactions. The planarity and non-planarity have been both observed for the FU pairs. According to the evaluated energies, the binding properties and also molecular orbital levels all reflect the degree of HB interactions in the paired systems. Electronic properties regarding the separation of HOMO and LUMO energy levels indicate the effects of $\mathrm{HB}$ types in the paired systems. For electron transferring, fluctuations in the favorability of receiving or releasing of one electron are observed for the investigated molecular pairs of FU. Interestingly, for anionic form, the non-favorability of singular FU to receive one electron is changed to favorability of receiving one electron in the FU pairs. According to the molecular orientations and the participating atoms in the HB interactions, the polarities of molecular systems are changed among the pairs as indicated by the values of dipole moments.

In addition to molecular properties, atomic scale properties like quadrupole coupling constants are also important. Among which, obtained magnitudes of $C_{\mathrm{Q}}$ (quadrupole coupling constants) could reveal insightful information about the electronic properties of matters at the atomic sites. The values of $C_{Q}$ for atoms of the investigated optimized models (Figs. 1-3) are summarized in Tables 3 and 4 . Since the electronic sites of atoms are perturbed during $\mathrm{HB}$ interactions, they could be well detected by the evaluated $C_{\mathrm{Q}}$ properties.

The first FU pair (Fig. 2, panel a) has only one type of $\mathrm{C}=\mathrm{O} \cdots \mathrm{H}-\mathrm{N}$ HB interactions, in which similar atoms are contributed to the intermolecular interactions. $\mathrm{H} 1$ and $\mathrm{O} 2$ are the major atoms directly related to HB interactions showing similar properties for two participating molecules. As it was mentioned earlier, the HB interactions are taken place because of electrons which were shared between the atoms. As could be seen from the magnitudes of $C_{\mathrm{Q}}, \mathrm{H} 1$ and $\mathrm{O} 2$ both lose their electrons in comparison with the parallel situation in the singular FU structures. Since the hydrogen atom has initial lack of electrons, the required amounts of electrons for $\mathrm{HB}$ interactions are provided by $\mathrm{N} 1$ atom as could be seen by a reduction of $C_{Q}$ for $N 1$ atom from singular FU to the first pair. Due to indirect effects of HB interactions on the properties of heterocyclic ring, the status of electronic distribution in the ring is changed as could be seen by changes of bond orders in the figures. Parallel to this trend, the $C_{\mathrm{Q}}$ 's are also changed for those atoms not directly participated in the $\mathrm{HB}$ interactions. In fact, there are no restrictions for electronic distributions in the chemical structures and they could be influenced by any perturbations on the original electronic distributions especially in the HB interacting systems. Among the atoms, since hydrogen atoms have actually lack of electrons, the changes of their properties because of indirect effects are almost negligible in comparison with other atoms. The lone pairs of electrons for nitrogen, oxygen, and fluorine atoms make them proper sites for detection of changes to electronic distributions by indirect effects of HB interactions as could be seen by comparisons of magnitudes for $C_{\mathrm{Q}}$ properties in the singular FU and molecular pairs. In the first molecular pair, the two counterparts are in the same geometries as the participating atoms to HB interactions are $\mathrm{H} 1$ and $\mathrm{O} 2$. For the second pair (Fig. 2, panel b), $\mathrm{O} 2$ atoms of both molecules contribute to $\mathrm{HB}$ interactions but $\mathrm{H3}$ and $\mathrm{H} 1$ of molecules number 3 and 4 participate in the interactions. Therefore, similar properties are not observed for the atoms of counter-parting molecules. In comparison with the first pair, the intermolecular distances are longer for the second one and smaller magnitude of EB has been also observed in the earlier section. Accordingly, smaller changes of magnitudes for $C_{Q}$ of $\mathrm{O} 2$ atoms have been observed for the second pair in comparison with the first pair. The properties for $\mathrm{H} 3$ and $\mathrm{H} 1$ of molecules FU3 and FU4 detect the direct effects of $\mathrm{HB}$ interactions whereas those of $\mathrm{H} 1$ and $\mathrm{H} 3$ of the same molecules are kept almost unchanged. As mentioned earlier, the hydrogen plays as a bridge in the HB interactions original bonded atom to $\mathrm{H}$ that is still very important as could be seen here for N3 and N1 atoms of FU3 and FU4 respectively. The properties for N3 and N1 are significantly reduced in comparison with the original singular FU, meaning the supporting role of nitrogen atoms to provide electrons for sharing in the HB interactions. The changes for N1 and N3 in the same molecules of FU3 and FU4 are not significant as much as the changes for participating $\mathrm{N}$ atoms in the HB interactions. Interestingly, it seems that the electron is induced in the site of $\mathrm{O} 4$ of FU3 as could be seen by increasing of the $C_{\mathrm{Q}}$ properties for this atom in the second pair regarding the lone molecule.

One important disadvantage of $\mathrm{FU}$ is its unwanted side effects for patients in the cancer therapies. Up to now, the results of the two pairs of FU indicate that the properties of each of FU pairs are not similar together, in which the properties for $\mathrm{O} 4$ atom is drastically induced in the second pair. Moreover, other atoms, which are not participated in the HB interactions, show the effects of geometrical variations in their electronic properties. The fluorine atom does not participate in $\mathrm{HB}$ interactions in the first set of pairs, but still shows the indirect effects of changes of electronic distributions in the heterocyclic ring because of HB interactions. In the real systems too many molecules are always gathered together; therefore, careful examinations of details of these gathering could be important due to their representing electronic properties as could be seen by the investigated pairs of FU. If the natures of molecules could be well determined, their corresponding behaviors and properties might be manageable. In the third FU pair of the first set, FU5 and FU6 interact together through $\mathrm{HB}$ interactions between $\mathrm{O} 2$ and $\mathrm{H} 3$ atoms. Similar atoms of the two molecules interact together in this pair like the first FU pair but here $\mathrm{H} 3$ is participated instead of $\mathrm{H} 1$ of first pair. Accordingly, N3 affected significantly by HB interactions due to contribution of $\mathrm{H} 3$ in the intermolecular interactions. Interestingly, the properties of two molecules are similar to each other and the major effects are observed for the properties of $\mathrm{N} 3, \mathrm{H} 3$, and $\mathrm{O} 2$ atoms in comparison with the evident molecule of singular FU. The indirect effects are still observed for other molecules especially for $\mathrm{C} 2$ atom which is existed in the interaction line as connected to the $\mathrm{O} 2$ atoms. The changes of distributions of electrons in the chemical structures of investigated models could be seen by the dash lines besides the covalent bonds, which are diverse for different bonds. The fourth FU pair (Fig. 2, panel d), includes FU7 and FU8 molecules, which interact together through 
$\mathrm{H} 3 \cdots \mathrm{O} 2$ and $\mathrm{O} 4 \cdots \mathrm{H} 1$ interactions. The conditions of molecules for interactions are different and the intermolecular distances are something similar to the first FU pair. The atoms $\mathrm{H} 3$ and $\mathrm{O} 4$ of FU7 and the atoms $\mathrm{H} 1$ and $\mathrm{O} 2$ of FU8 are in the frontiers of $\mathrm{HB}$ interactions, in which their properties accordingly are affected significantly. Two carbon atoms of both molecules are influenced considerably compared to the previous paired models as could be seen by their magnitudes of $C_{\mathrm{Q}}$. Interestingly, the effects for $\mathrm{F}$ atoms are different from what was seen for previous models. The trends again insist on the importance of geometrical properties on the electronic responses of the corresponding structures. The atoms N3 of FU7 and N1 of FU8 play the roles of electronic supporting for their $\mathrm{H}$ atoms to participate in the $\mathrm{HB}$ interactions and the changes of their properties are significant in comparison with the singular FU. However, the changes for N1 of FU7 and N3 of FU8 are less significant in the investigated pair. The oxygen atom is always a good atomic site for contributing to possible HB interactions with other atoms and molecules and it plays a dominant role in this case. The results indicate that the properties for $\mathrm{O} 2$ atom are drastically changed by the inductions of electrons in this atomic site of FU7.

It is important to note that inductions of electrons in the atomic sites could increase the reactivity of that atom because of their different interactions with other molecules or atoms; hence, the side effects might rise. The molecules FU9 and FU10 belong to the fifth FU pair, in which $\mathrm{H} 3$ and $\mathrm{O} 4$ of FU9 and $\mathrm{H} 3$ and $\mathrm{O} 2$ of FU10 are directly participated in the $\mathrm{HB}$ interactions. According to the obtained $C_{\mathrm{Q}}$, the properties for $\mathrm{N} 3$ of both molecules are significantly changed due to their supporting role for $\mathrm{H}$ atoms. The properties for $\mathrm{O} 4$ and $\mathrm{O} 2$ of FU9 and FU10 are also changed due to direct effects of HB interactions. Within the current FU pair, the properties for $\mathrm{O} 2$ and $\mathrm{O} 4$ of FU9 and FU10 show the abnormal properties of electron inductions in their atomic sites. Interestingly, this pair is the most unstable one according to the binding energies among the first set of FU pairs, in which the magnitudes for $C_{\mathrm{Q}}$ properties of oxygen atoms also reveal high reactivity for this one. For the sixth FU pair of the first set (Fig. 2, panel f), the atomic sites of two molecules are similar for the HB interactions; H3 and O4. Parallel to the results of the first and third pairs of Fig. 2, panels (a) and (c), the properties of atoms are expected to be similar for two molecular counterparts. For these types of similar FU pairs, the intermolecular distances thorough two HB interacting atomic sites are completely in the same magnitudes showing similarities of properties for the molecular counterparts of each investigated model. Moreover, the magnitudes of dipole moments are almost zero for the similar FU pairs showing a kind of electronic distribution in the paired molecular system. In the sixth FU pair, $\mathrm{O} 4$ is contributed to the HB interactions and the electron induction is still observed for the $\mathrm{O} 2$ atoms in both molecules, in which the magnitudes of $C_{\mathrm{Q}}$ properties are increased in the FU pair regarding the singular FU. The properties of other atoms and also fluorine are also changed due to the indirect effects of HB interactions. As concluding remarks for the atomic properties of the first set of FU pairs, trends could be listed as follows: According to the geometrical properties, if similar atoms of two molecules interact with each other, the intermolecular distance of two interacting sites are the same. According to this geometrical similarity, the atomic properties of atoms are similar at the corresponding positions according to the equal magnitudes of $C_{\mathrm{Q}}$ properties for each atomic type. Interestingly, the effects of $\mathrm{HB}$ interactions are observed for all atomic structures but emphasized for the directly interacting atoms. Moreover, the electron is induced for non-interacting oxygen atom, which could make the structures more reactive but less stable. It is important to note that there are two types of oxygen atoms in the FU structure, $\mathrm{O} 2$ as urea type and 04 as amide type. Therefore, the properties of these two oxygen atoms are initially different according to their own types. In fact, the different distributions of electrons separate the two types of oxygen atoms and their behaviors will be also characteristic for each one. Nitrogen atoms have supporting role for hydrogen atoms for contributing to HB interactions and they detect remarkable effects. The carbon atoms and also the fluorine atoms which are away from the HB interactions regions also detect the effects by the HB interactions. And finally, the molecular properties such as dipole moment could be explained by the atomic properties as could be seen by the zeromagnitude of dipole moment for the FU pairs with similar atomic properties for atoms of each counterpart.

The second set of FU pairs (Fig. 3) include C-F..H-C, $\mathrm{C}-\mathrm{F} \cdots \mathrm{H}-\mathrm{N}$, and $\mathrm{C}-\mathrm{H} \cdots \mathrm{O}=\mathrm{C}$ types of $\mathrm{HB}$ interactions in addition to the only $\mathrm{C}=\mathrm{O} \cdots \mathrm{H}-\mathrm{N}$ type of $\mathrm{HB}$ interactions for the first set. The results are presented in Tables 3 and 4 and the figures are showed in the panels g-k of Fig. 3. It is worth noting that, the strength of $\mathrm{HB}$ interactions for the second set of FU pairs are weaker than the first set according to their intermolecular geometries and binding energies. Parallel to the results of geometries and energies, the changes of magnitude for $C_{\mathrm{Q}}$ properties in the $\mathrm{HB}$ interactions are expected to be smaller than those of the first set. FU13 and FU14 belong to the first pair of the second set (Fig. 3, panel $\mathrm{g}$ ), which are interacting each other through strong $\mathrm{C}=\mathrm{O} \cdots \mathrm{H}-\mathrm{N}$ and weak $\mathrm{C}-\mathrm{F} \cdots \mathrm{H}-\mathrm{C}$ types of $\mathrm{HB}$ interactions. The changes of $C_{Q}$ properties for interacting 04 and $\mathrm{H} 1$ atoms show the effects of strong $\mathrm{HB}$ interactions but the properties for $\mathrm{F}$ and $\mathrm{H} 6$ atoms indicate that the interactions are weak. The properties for $\mathrm{N} 1$ of FU13 also confirm strong $\mathrm{HB}$ interactions for $\mathrm{C}=\mathrm{O} \cdots \mathrm{H}-\mathrm{N}$ interaction. Here the changes of $C_{Q}$ properties for $\mathrm{F}$ and $\mathrm{H} 6$ atoms are small but important for the weak HB interactions. It is important to note that the electronic properties for atomic counterparts of weak HB interactions are slightly changed and they could not be easily measured, in which quantum computations could yield insightful information for them. The second pair of the second set involves FU15 and FU16 (Fig. 3, panel h), in which the changes for $C_{\mathrm{Q}}$ properties of $\mathrm{H} 6$ of FU15 and $\mathrm{H} 1$ of FU16 show that the HB interactions are strong enough to be well considered. Interestingly, $\mathrm{C}-\mathrm{H} 6 \ldots \mathrm{O} 2=\mathrm{C}$ is a type of non-classical interactions but with notable effects on electronic properties. Interestingly, the changes for $\mathrm{F}$ atom of FU15 indicate that the $\mathrm{C}-\mathrm{F} \cdots \mathrm{H}-\mathrm{N}$ is very well established in this FU pair. The strength of $\mathrm{C}-\mathrm{F} \cdots \mathrm{H}-\mathrm{N}$ interaction is less than $\mathrm{C}=\mathrm{O} \cdots \mathrm{H}-\mathrm{N}$ interaction but the changes of magnitudes for $C_{\mathrm{Q}}$ properties indicate that the interaction is important. The third pair of the second set (Fig. 3, panel i), includes $\mathrm{C}-\mathrm{F} \cdots \mathrm{H}-\mathrm{N}$ and $\mathrm{C}-\mathrm{H} \cdots \mathrm{O}=\mathrm{C}$ interactions, which are both important according to their observed effects by changes of their $C_{\mathrm{Q}}$ properties from the original singular FU to this pair.

The $\mathrm{HB}$ type of $\mathrm{C}-\mathrm{H} \cdots \mathrm{O}=\mathrm{C}$ interaction is very important because of its significant changes of properties. Here, it is again observed that the $\mathrm{C}-\mathrm{F} \cdots \mathrm{H}-\mathrm{N}$ bond is weaker that the $\mathrm{C}=\mathrm{O} \cdots \mathrm{H}-\mathrm{N}$ one but still strong enough to keep the molecules together in an appropriate distance. In the next FU pair (Fig. 3, panel j), the situation is something similar to the last FU pair but 04 contributes to $\mathrm{HB}$ interactions instead of $\mathrm{O} 2$ of the last pair. By the obtained results, it could be understood that the role of $\mathrm{H} 6$ is dominant for the HB interactions among the investigated FU pairs. Since H6 is considered as a contributor to weak HB interactions, revealing the changes of its properties by the correct determinations of intermolecular interactions is very important. The final FU pair (Fig. 3, panel $\mathrm{k}$ ), includes the similar atomic sites for contributing to weak $\mathrm{HB}$ interactions of $\mathrm{C}-\mathrm{F} \cdots \mathrm{H}-\mathrm{C}$ types. It seems that the $\mathrm{F}$ atom attracts the shared electrons to its own side as could be seen by the increased value of $C_{Q}$ in comparison with the singular FU molecule. As concluding remarks of the second set of FU pairs, it could be mentioned that effects of interactions reflected less on the atomic properties in comparison to the first set of FU pairs. The 
weak $\mathrm{HB}$ interactions are taken place due to the dominant role of $\mathrm{H} 6$ atom through $\mathrm{C}-\mathrm{H}$ counterpart for $\mathrm{HB}$ interactions. The planarity of FU pair could be perturbed as the $\mathrm{O}$ and $\mathrm{F}$ see each other in a close distance and it causes molecular repulsion to find a better intermolecular geometry. The electronic properties for noninteracting oxygen atom is still induced but is less than the induction as was seen in the first set of FU pairs. The properties of other non-interacting atoms could also be affected by the indirect effects of $\mathrm{HB}$ interactions but slight changes due to longer distance of intermolecular positions in the second set in comparison with the first set. The weak HB interactions are well established in the FU pairs, which are very well influenced by the changes of $C_{Q}$ atomic properties.

\section{Conclusions}

We have performed DFT calculations on all possible structures of FU pairs to investigate various types of $\mathrm{HB}$ interactions for these systems. The obtained results by optimizations indicated that the model structures could have different shapes according to their interactions situations, but are in planar systems. Moreover, electron transferring properties are also changed from the singular FU to paired molecules. The results indicated that the atomic $C_{\mathrm{Q}}$ properties could very well detect the effects of $\mathrm{HB}$ interactions and they could also determine the strengths of $\mathrm{HBs}$ as weak or strong ones. The roles of $\mathrm{H}$ atoms in the $\mathrm{HB}$ interactions to connect the molecules together and also weak HB interactions are very well investigated. And finally, although the regular types of HB interactions are always important to be investigated, but irregular ones are also important due to their activities in determining the structural forms of base pairs.

\section{Acknowledgement}

M.M. acknowledges the kind helps of Mr. S. Fazel, professional teacher of English, for his efforts to revise the English of this manuscript.

\section{References}

[1] T. Fornaro, D. Burini, M. Biczysko, V. Barone, Hydrogen-bonding effects on infrared spectra from anharmonic computations: uracil-water complexes and uracil dimers, J. Phys. Chem. A 119 (2015) 4224-4236.

[2] R. Ida, M.D. Clerk, G. Wu, Influence of $\mathrm{N}-\mathrm{H} \ldots \mathrm{O}$ and $\mathrm{C}-\mathrm{H} \ldots \mathrm{O}$ hydrogen bonds on the 170 NMR tensors in crystalline uracil: Computational study, J. Phys. Chem. A 110 (2006) 1065-1071.

[3] W.S. Koski, The hydrogen bond, J. Am. Chem. Soc. 82 (1960) 4120.

[4] K. Ruiz-Mirazo, C. Briones, A. Escosura, Prebiotic systems chemistry: new perspectives for the origins of life, Chem. Rev. 114 (2014) 285-366.

[5] X. Ma, X. Lv, N. Qiu, B. Yang, Q. He, Y. Hu, Discovery of novel quinoline-based mTOR inhibitors via introducing intra-molecular hydrogen bonding scaffold (iMHBS): the design, synthesis and biological evaluation, Bioorg. Med. Chem. 23 (2015) 7585-7596.

[6] V.V. Zhurov, A.A. Pinkerton, Inter- and intramolecular interactions in crystalline 2-nitrobenzoic acid - an experimental and theoretical QTAIM analysis, J. Phys. Chem. A 119 (2015) 13092-13100.

[7] R. Moreno-Fuquen, J.C. Castillo, D. Becerra, H. Camargo, J.A. Henao, Crysta structure of 2-fluoro-n-(1,3-thia-zol-2-yl)benzamide, Acta Cryst. E 71 (2015) 0882-0883.

[8] M. Solimannejad, M. Malekani, I. Alkorta, Substituent effects on the cooperativity of halogen bonding, J. Phys. Chem. A 117 (2013) 5551-5557.

[9] M. Mirzaei, N.L. Hadipour, An investigation of hydrogen-bonding effects on the nitrogen and hydrogen electric field gradient and chemical shielding tensors in the 9-methyladenine real crystalline structure: a density functional theory study, J. Phys. Chem. A 110 (2006) 4833-4838.

[10] R.E.A. Kelly, Y.J. Lee, L.N. Kantorovich, Homo pairing possibilities of the DNA bases cytosine and guanine: an ab initio DFT study, J. Phys. Chem. B 109 (2005) 22045-22052.

[11] A. Mohajeri, F.F. Nobandegani, Detection and evaluation of hydrogen bond strength in nucleic acid base pairs, J. Phys. Chem. A 112 (2008) 281-295.
[12] A. Diez-Martinez, E.K. Kim, R. Krishnamurthy, Hydrogen-bonding complexes of 5-azauracil and uracil derivatives in organic medium, J. Org. Chem. 80 (2015) 7066-7075.

[13] M. Mirzaei, N.L. Hadipour, Study of hydrogen bonds in crystalline 5nitrouracil. Density functional theory calculations of the $0-17, \mathrm{~N}-14$, and $\mathrm{H}-$ 2 nuclear quadrupole resonance parameters, J. Iran. Chem. Soc. 6 (2009) 195199.

[14] M. Mirzaei, N.L. Hadipour, Study of hydrogen bonds in 1-methyluracil by DFT calculations of oxygen, nitrogen, and hydrogen quadrupole coupling constants and isotropic chemical shifts, Chem. Phys. Lett. 438 (2007) 304-307.

[15] B.N. Bourdélat-Parks, R.M. Wartell, Thermodynamics of RNA duplexes with tandem mismatches containing a uracil-uracil pair flanked by C.G/G.C or G.Cl A.U closing base pairs, Biochemistry 44 (44) (2005) 16710-16717.

[16] M. Solimannejad, S. Scheiner, Stabilities and properties of complexes pairing hydroperoxyl radical with monohalomethanes, J. Phys. Chem. A 110 (2006) 5948-5951.

[17] T. Fornaro, I. Carnimeo, M. Biczysko, Toward feasible and comprehensive computational protocol for simulation of the spectroscopic properties of large molecular systems: the anharmonic infrared spectrum of uracil in the solid state by the reduced dimensionality/hybrid VPT2 approach, J. Phys. Chem. A 119 (2015) 5313-5326.

[18] T. Partovi, M. Mirzaei, N.L. Hadipour, The $\mathrm{C}-\mathrm{H}$... O hydrogen bonding effects on the ${ }^{17} \mathrm{O}$ electric field gradient and chemical shielding tensors in crystalline 1methyluracil: a DFT study, Z. Naturforsch. A 61 (2006) 383-388.

[19] S. Wang, X.H. Ding, Y.H. Li, W. Huang, Effects of strong hydrogen bonds and weak intermolecular interactions on supramolecular assemblies of 4fluorobenzylamine, J. Mol. Struct. 1091 (2015) 98-108.

[20] M. Mirzaei, N.L. Hadipour, A computational NQR study on the hydrogenbonded lattice of cytosine-5-acetic acid, J. Comput. Chem. 29 (2008) 832-838.

[21] G.R. Medders, F. Paesani, Infrared and Raman spectroscopy of liquid water through "first-principles" many-body molecular dynamics, J. Chem. Theor. Comput. 11 (2015) 1145-1154.

[22] J. Ho, M.B. Newcomer, C.M. Ragain, J.A. Gascon, E.R. Batista, J.P. Loria, V.S. Batista, MoD-QM/MM structural refinement method: characterization of hydrogen bonding in the oxytricha nova G-quadruplex, J. Chem. Theor. Comput. 10 (2014) 5125-5135.

[23] L. Fallon III, The crystal and molecular structure of 5-fluorouracil, Acta Cryst. B 29 (1973) 2549-2556.

[24] C. Marin-Vicente, Y. Lyutvinskiy, P.R. Fuertes, R.A. Zubarev, N. Visa, The effects of 5-fluorouracil on the proteome of colon cancer cells, J. Proteome Res. 12 (2013) 1969-1979.

[25] S. Zhan, Q. Zhao, S. Chen, J. Wang, Z. Liu, C. Chen, Solubility and partition coefficients of 5-fluorouracil in $\mathrm{ScCO}_{2}$ and $\mathrm{ScCO}_{2} /$ poly(l-lactic acid), J. Chem. Eng. Data 59 (2014) 1158-1164.

[26] W. Liu, X. Li, Y.S. Wong, W. Zheng, Y. Zhang, W. Cao, T. Chen, Selenium nanoparticles as a carrier of 5-fluorouracil to achieve anticancer synergism, ACS Nano 6 (2012) 6578-6591.

[27] A.L. LaFrate, J.A. Katzenellenbogen, Improved chemical syntheses of 5,6dihydro-5-fluorouracil, J. Org. Chem. 72 (2007) 8573-8576.

[28] M.J. Frisch, G.W. Trucks, H.B. Schlegel, G.E. Scuseria, M.A. Robb, et al., Gaussian 98, Revision A.7, Gaussian Inc.: Pittsburgh, PA, 1998.

[29] M. Mirzaei, F. Elmi, N.L. Hadipour, A systematic investigation of hydrogenbonding effects on the $170,14 \mathrm{~N}$, and $2 \mathrm{H}$ nuclear quadrupole resonance parameters of anhydrous and monohydrated cytosine crystalline structures: a density functional theory study, J. Phys. Chem. B 110 (2006) 10991-10996.

[30] S.F. Boys, F. Bernardi, The calculation of small molecular interactions by the differences of separate total energies. Some procedures with reduced errors, Mol. Phys. 19 (1970) 553-566.

[31] T.P. Das, E.L. Han, Nuclear Quadrupole Resonance Spectroscopy, Academic Press, New York, 1958.

[32] P. Pyykkö, Spectroscopic nuclear quadrupole moments, Mol. Phys. 99 (2001) 1617-1629.

[33] L.G. Butler, T.L. Brown, Nuclear quadrupole coupling constants and hydrogen bonding. Molecular orbital study of oxygen-17 and deuterium field gradients in formaldehyde-water hydrogen bonding, J. Am. Chem. Soc. 103 (1981) $6541-$ 6549.

[34] M. Mirzaei, N.L. Hadipour, M.R. Abolhassani, Influence of C-doping on the B-11 and $\mathrm{N}-14$ quadrupole coupling constants in boron-nitride nanotubes: a DFT study, Z. Naturforsch. A 62 (2007) 56-60.

[35] C. Puzzarini, C. Cabezas, M. Biczysko, J.L. Alonso, V. Barone, I. Pena, Accurate molecular structure and spectroscopic properties of nucleobases: a combined computational-microwave investigation of 2-thiouracil as a case study, Phys. Chem. Chem. Phys. 15 (2013) 16965-16975.

[36] C. Kamal, A. Banerjee, A. Chakrabarti, T.K. Ghanty, Interesting periodic variations in physical and chemical properties of homonuclear diatomic molecules, Int. J. Quant. Chem. 112 (2012) 1097-1106.

[37] C. Puzzarini, V. Barone, Extending the molecular size in accurate quantumchemical calculations: the equilibrium structure and spectroscopic properties of uracil, Phys. Chem. Chem. Phys. 13 (2011) 7158-7166.

[38] V. Vaquero, M.E. Sanz, J.C. Lopez, J.L. Alonso, The structure of uracil: a laser ablation rotational study, J. Phys. Chem. A 111 (2007) 3443-3445.

[39] K. Harismah, O.M. Ozkendir, M. Mirzaei, Explorations of crystalline effects on 4-(benzyloxy)benzaldehyde properties, Z. Naturforsch. 70 (2015) 1013-1018. 